\title{
DISERTACIONES
}

ESTUDIOS

\section{UNA GEOGRAFÍA DE MÉXICO EN NETFLIX: RECOMENDACIONES AUTOMATIZADAS Y DIVERSIDAD DE CONTENIDOS OFERTADOS*}

\section{A Netflix Geography of Mexico: Automatized Recommendations and Content Supplied Diversity}

\section{Uma geografia do México na Netflix: recomendações automatizadas e diversidade de conteúdo oferecido} Gabriela Elisa Sued, Instituto de Investigaciones Sociales de la Universidad Nacional Autó-
noma de México (México)

gabriela.sued@sociales.unam.mx

\author{
Recibido: 24 de mayo del 2021 \\ Aprobado: 05 de julio del 2021 \\ Fecha de prepublicación: 15 de octubre del 2021
}

\section{RESUMEN}

Se presenta un estudio centrado en información brindada por usuarios sobre las lógicas automatizadas de recomendación de contenidos asociados a México en Netflix. Se propone determinar si estos contribuyen a la diversidad audiovisual, así como identificar los parámetros de la plataforma para efectuar recomendaciones. Se diseña y aplica un instrumento de indagación digital basado en técnicas de auditoría algorítmica. Se comprueba que hay fuentes que se priorizan y que son de origen norteamericano. Se concluye que los contenidos son poco diversos

* Becaria Postdoctoral Conacyt. La autora agradece al programa Estancias Postdoctorales por México 20202021 el financiamiento para la realización de este artículo. 


\title{
DISERTACIONES
}

y se orientan según el género - femenino o masculino- de los usuarios, que la personalización es baja y que la audiencia presenta una alta aceptación a las recomendaciones, excepto en tematizaciones del narcotráfico y estereotipos de género. En consecuencia, se abre una posibilidad de intervención en la plataforma, a través de las recomendaciones, para tratar la diversidad.

Palabras clave: recomendaciones automatizadas; diversidad audiovisual; México; Netflix; audiencias.

\begin{abstract}
This study focuses on the information provided by users to the automated logic of Netflix concerning the Mexican-associated contents it suggests. It aims to define whether these contribute to audiovisual diversity and identify the parameters that the platform considers when making recommendations. We designed and applied a digital research tool based on algorithmic auditing techniques, and evidence is found that certain priority is given to some sources and that these are North American. Thus, we conclude that there is little variety in suggestions and that they are gender-oriented. Further, low personalization has been observed, and the audience has been found to widely accept the recommendations, except in the case of themes related to drug trafficking and female gender stereotypes. Consequently, a possibility of intervention in the platform is opened, through the recommendations, to deal with diversity.
\end{abstract}

Keywords: Automatic recommendations; audiovisual diversity; Mexico; Netflix; audience.

\section{RESUMO}

É apresentado um estudo com foco nas informações fornecidas pelos usuários sobre a lógica automatizada de recomendação de conteúdo associada ao México na Netflix. Propõe-se verificar se contribuem para a diversidade audiovisual, bem como identificar parâmetros que a plataforma leva em consideração para fazer recomendações. Se desenha e aplica um instrumento de indagação digital baseado em técnicas de auditoria algorítmica. Se comprova que há fontes priorizadas e que são de origem norte-americana. Conclui-se que os conteúdos não são muito diversos e se orientam de acordo com o gênero feminino ou masculino dos usuários, que a personalização é baixa e que o público apresenta alta aceitação das recomendações, exceto nas tematizações do narcotráfico e dos estereótipos de gênero feminino. Em consequência, se abre uma possibilidade de intervenção na plataforma, através das recomendações, para tratar a diversidade.

Palavras-chave: recomendações automatizadas; diversidade audiovisual; México; Netflix; audiências. 
En el contexto de una creciente migración de los contenidos televisivos hacia las plataformas digitales de video bajo demanda (Piñón, 2020), las decisiones de las audiencias se encuentran mediadas por sistemas de recomendación automatizados que orientan sus preferencias. Netflix es un caso típico donde la mediación algorítmica resulta necesaria para la gestión de su catálogo y el acceso a contenidos por parte de sus clientes.

Con presencia en 190 países, Netflix es una empresa transnacional tanto como una empresa local. Su transformación de compañía nacional norteamericana a empresa global mundial se basa en una estrategia de transnacionalización desplegada en múltiples niveles. La plataforma tuvo que adaptarse a las regulaciones de los medios, los gustos locales y las preferencias de la audiencia en cada país donde tiene presencia (Lobato, 2019). Seguida de la estrategia de transnacionalización que garantiza su presencia a nivel mundial, se presenta la de personalización, que asegura la preferencia y permanencia de su audiencia frente a sus competidores en el servicio de video bajo demanda. Según se lee en uno de los sitios web de la compañía: "la personalización es uno de los pilares de Netflix, porque permite a cada miembro tener una visión diferente de nuestros contenidos que se adapta a sus intereses y puede ayudar a ampliarlos" ${ }^{1}$ (Netflix Research, s.f.). Esta estrategia centrada en los gustos del cliente podría impactar positivamente en términos de variedad de contenidos, dada la diversidad de la audiencia a la que se apunta (Ranavoison, 2019).

Este estudio aborda las recomendaciones acerca de México en Netflix. Se propone determinar si las recomendaciones ofrecidas contribuyen a la lógica diversa que la plataforma pregona como política, así como determinar los parámetros que la plataforma tiene en cuenta para efectuar sus recomendaciones.

Los propósitos del trabajo se traducen en las siguientes preguntas de investigación: ¿responden las recomendaciones automatizadas acerca de México a una lógica de diversidad audiovisual? ¿Qué tipo de información de entrada toman en cuenta los sistemas de recomendación para sugerir contenidos? ¿Cuál es la aceptación de los usuarios de los contenidos recomendados por la plataforma? Las respuestas a estas preguntas nos permitirán, además, visualizar a México en Netflix como un espacio producido a través de prácticas mediáticas de selección y jerarquización de contenidos en el que intervienen tanto elementos transnacionales, como locales (Padilla de la Torre, 2012).

\section{Antecedentes}

\section{Los algoritmos de Netflix y la diversidad audiovisual}

Los sistemas automatizados de recomendación sugieren y proveen a los usuarios información que ellos predicen les será relevante. Han sido diseñados para que las plataformas sociales como Facebook, YouTube, Netflix y Spotify, entre otras, resuelvan problemas de jerarquización, filtrado y organización de información eficientemente (Bucher, 2018). En el caso de Netflix, por un lado, forman parte del valor diferencial que la compañía ofrece a sus clientes y, por el otro, constituyen una de las vías de acceso de los usuarios a los contenidos (Gómez-Uribe \& Hunt, 2016).

Debido a sus tareas autónomas de selección y jerarquización, los algoritmos de recomendación asumen un rol importante en la promoción de contenidos diversos. A la vez, la revisión, filtrado y jerarquización de un catálogo

1 Traducción propia. 


\section{DISERTACIONES}

ESTUDIOS

Economía de la a ter

ssv: 1856-9536

Doi: https://doi.org/10.12804/revistas.urosario.edu.co/disertaciones/a.10616

Volumen 15, Número 2/ Julio-diciembre 2022

Versión pof para imprimir desde

http://revistas.urosario.edu.co/index.php/disertaciones

nacional puede decir mucho sobre los contenidos que Netflix propone en función de las audiencias de cada país (Lobato, 2017). Los algoritmos de recomendación no se formulan en aislamiento, sino que forman parte de un sistema que incluye infraestructuras, datos e interfaces y se enmarcan en un conjunto de formas de conocimiento, legalidades y cuestiones financieras (Rieder, 2020). Desde luego, esta complejidad de factores sumada a la reticencia de las plataformas de hacer públicos los principios de sus sistemas de búsqueda y recomendación imposibilitan develar el funcionamiento de los algoritmos de la plataforma en una suerte de ingeniería reversa. No obstante, los patrones emergentes de la información proporcionada por los usuarios pueden contribuir a la identificación de ciertas regularidades que indiquen los valores culturales, demográficos y empresariales incluidos en ellos.

El algoritmo de búsqueda que se estudia en este trabajo difiere del sistema de recomendaciones que se despliega cuando el usuario accede a la página de inicio de Netflix. Mientras que el primero es una interfaz pull que implica que el usuario tome la iniciativa en la búsqueda de información y el sistema la entregue, el segundo es una interfaz push, en la que la plataforma se encarga de ofrecer información procesada, organizada y elaborada (Cybenko \& Brewington, 1999). Según Netflix, la búsqueda es una experiencia personalizada que lleva a los usuarios a descubrir nueva información: "Sabemos que a veces los miembros tienen algo en mente cuando visitan nuestro servicio, así que proveemos una capacidad sofisticada de búsqueda para ofrecer los videos adecuados a nuestros miembros"2 (Netflix Research, s. f.). Para Jenner (2018), el algoritmo de Netflix no es tan personalizado como lo describe la empresa ni tan prescriptivo como una grilla televisiva, pero empuja a los usuarios a privilegiar unos contenidos sobre otros.

Uno de los problemas que se reconoce en estos asistentes de búsqueda automatizada es el de la amplificación algorítmica. Sucede cuando los usuarios seleccionan una de las opciones ofrecidas y luego estas se transforman en nuevo conocimiento para entrenar el algoritmo, creando un bucle o ciclo de retroalimentación en el que la salida del algoritmo se convierte en parte de su entrada. En consecuencia, los algoritmos suelen realizar recomendaciones similares a las elecciones realizadas, reforzando la popularidad del contenido que ya es popular (Sadagopan, 2019), comportamiento que puede afectar a la recomendación de contenidos diversos. Una variante de este comportamiento es el identificado por Tufekci (2018): se produce cuando luego de una versión liviana de un contenido se provee una recomendación de esa misma temática pero más acentuada. La autora pone como ejemplo que videos relacionados con el supremacismo blanco le fueran ofrecidos luego de ver algunos videos de Donald Trump a fin de estudiar su discurso.

Por otra parte, la diversidad audiovisual es un objetivo deseable tanto en el sistema de medios mexicano como en el mundial, dado que las audiencias no pueden considerarse homogéneas ni portadoras de los mismos valores sociales y culturales (Lozano, 2006). Según García Leiva (2019), la diversidad en la industria audiovisual depende de tres aspectos: fuentes, contenidos y exposición. La diversidad de fuentes refiere a la propiedad de las productoras y distribuidoras de contenidos y a la conformación de su fuerza de trabajo. La diversidad de contenidos incluye la variedad en los formatos, la representación demográfica y las ideas. La diversidad en la exposición de las audiencias comprende la exposición horizontal, entendida como la fragmentación de las audiencias entre todos los contenidos disponibles, y la exposición vertical, que comprende patrones individuales de consumo (Albornoz \& García Leiva,

2 Traducción propia. 
2017). Ranavoison (2019) agrega a estas variables las diversidades ofertada y consumida. La primera considera la diversidad ofertada por los medios y la segunda la que de hecho es consumida por las audiencias.

La diversidad de fuentes se hace más difícil de identificar. En el caso de México, varias producciones que presentan narrativas y actores mexicanos son producidas por compañías estadounidenses (Orozco Gómez, 2020). Esta relación dista de ser equitativa. En el campo audiovisual, la asimetría en términos de difusión de producciones propias e inversión extranjera es histórica y ampliamente favorable a Estados Unidos, ya desde antes de las firmas de los primeros tratados comerciales entre ambos países (García Canclini, 1996).

La diversidad de contenido debe incorporar a la variedad, las listas automatizadas de recomendaciones que priorizan unos contenidos por sobre otros donde no todos tienen el mismo valor: están los primeros de la lista, de más fácil acceso y por ende concentradores de mayor atención, y también los últimos, de visibilidad menor. Por otro lado, a diferencia de las grillas televisivas, las clasificaciones son variables y producto de factores no del todo conocidos ni cognoscibles (Rieder et al., 2018). La diversidad horizontal, por otra parte, emerge de procesos algorítmicos opacos que involucran diversos factores que las empresas no dan a conocer, entre los que figuran el historial de visualizaciones de los usuarios, similitudes entre patrones de consumo (Plummer, 2017), medición de tiempos de visionado por usuarios, identificación de momentos del día en que se conectan a la plataforma, dispositivos utilizados y qué nuevos programas se eligen al terminar otros (Hallinan \& Striphas, 2016).

Hallinan y Striphas (2016) estudian las relaciones entre los algoritmos de Netflix y la producción cultural, asumiendo que los primeros producen sentido sobre la segunda. En dicho trabajo los autores estudian el caso del Premio Netflix, concurso organizado entre 2006 y 2009 por la compañía para mejorar su algoritmo de recomendación. En ese estudio, los autores observan cómo la cultura como conocimiento especializado se subsume a las razones técnicas de los especialistas informáticos, quienes dejaron de lado los criterios demográficos básicos para basarse en cálculos matemáticos realizados sobre la calificación que los usuarios realizan sobre los productos. Los autores estudian también cómo Netflix recolecta los consumos de los usuarios para identificar patrones que lleven al éxito de los contenidos producidos por la compañía, como sucede en el caso de House of Cards.

En un estudio cualitativo centrado en usuarios, Siles et al. (2019) observan las relaciones entre algoritmos y usuarios como un proceso de mutua domesticación, en el que la plataforma modela a sus usuarios ideales, pero a la vez los usuarios construyen su propio vínculo con la plataforma a partir de dinámicas de personalización, resistencias, rituales e integración de las recomendaciones automatizadas con sus propios criterios de selección de contenidos.

Heredia-Ruiz et al. (2021) estudian los flujos televisivos de Netflix a través del análisis de los estrenos de la plataforma desde 2019. Este estudio tiene implicancias para el presente, ya que los autores destacan, por un lado, la importancia de abordar la plataforma desde una perspectiva geográfica, a fin de determinar cuánto de global y cuánto de local posee el catálogo de cada país y, por el otro, resaltan la importancia de las producciones norteamericanas al destacar que de los estrenos estudiados, la mitad eran producciones norteamericanas. En este sentido, el trabajo de lordache (2021) sobre el catálogo de Netflix de cuatro países europeos resulta relevante, ya que la autora encuentra que cerca de la mitad del catálogo de estos países corresponde a compañías norteamericanas y a producciones donde predomina el idioma inglés, pese a la directiva de Servicios de Medios Audiovisuales de 2018, en la que se impuso una cuota de contenidos europeos del $30 \%$ a servicios de transmisión en directo -streaming- extranjeros. 


\section{Materiales y métodos}

Para contestar las preguntas de investigación se diseñó un instrumento de indagación digital basado en técnicas de auditoría algorítmica (Sandvig et al., 2014). Estas generan una situación simulada en un ambiente controlado a fin de generar datos de salida, por ejemplo: una lista de resultados. Esta lista se compara con un elemento de entrada, por ejemplo, la clave de búsqueda, de la que se deducen las variables que inducen la salida de datos. Bandy (2021) realiza una revisión de literatura de 62 artículos relevantes que aplican auditorías algorítmicas para diagnosticar conductas problemáticas en sistemas algorítmicos de plataformas como Google, YouTube, Booking, Twitter, Facebook y Spotify. Estas investigaciones ponen en juego diferentes estrategias de auditorías entre las que se encuentran la captura de datos digitales, la creación de identidades falsas para la ejecución de un programa o la colaboración entre usuarios, también denominada crowdsourcing -colaboración masiva voluntaria- para el testeo de un algoritmo. A partir de la revisión de estos trabajos, se identificaron cuatro categoría de comportamientos algorítmicos distorsionados: discriminación, distorsión, explotación y juicio erróneo. La más estudiada en relación con sistemas de búsqueda y recomendación es la distorsión por ser una manera de presentar resultados o recomendaciones de manera que se producen desvíos hacia información falsa, polarizaciones, cámaras de eco o falta de diversidad.

El protocolo diseñado para este trabajo fue contestado por 79 usuarios, quienes proveyeron la información necesaria para este estudio entre el 26 de octubre y el 11 de noviembre de 2020. El conjunto se compone de un $60 \%$ de mujeres y un $40 \%$ de varones, de un $90 \%$ de residentes en México y un $10 \%$ de residentes en España y Argentina, con edades que varían entre los 18 y los 67 años.

La muestra contiene tres tipos de información: títulos recomendados, información demográfica y opiniones y precisiones acerca de la aceptación de las recomendaciones efectuadas por el sistema. Para obtener la información sobre títulos recomendados se solicitó a los participantes impresiones de pantalla que recrearon una consulta en el buscador de Netflix mediante tres palabras clave: "México", "mexicanas" y "mexicanos". Los usuarios entraron en la plataforma Netflix con sus usuarios habituales para realizar estas consultas. En la selección de las consultas se apeló a cierta polisemia y significado abierto en los que México no solamente sería una parte del título de la recomendación, sino también la constitución de un espacio de producción, narración y referencia. Esta técnica abrió una vía de acceso directo a las recomendaciones que la plataforma ofrece. Los datos demográficos incluyeron información de género, rango de edad y lugar de residencia. Para conocer las opiniones de los consultados sobre las recomendaciones, se formularon preguntas abiertas y cerradas, previo procesamiento de las impresiones de pantalla enviadas. Entre el primer grupo, se preguntó a los consultados si asociaban la lista de recomendaciones obtenida con sus consumos. Entre el segundo grupo, se inquirió si compartían su perfil con otras personas y si verían o estarían dispuestos a ver un conjunto de títulos seleccionados entre los resultados más frecuentes de las listas obtenidas.

Esta estrategia se complementó con la revisión del sitio de recomendaciones Reelgood.com. Este sitio contiene una base de datos con el catálogo de Netflix disponible en Internet donde resulta posible realizar búsquedas por país. La información allí disponible fue útil para identificar la cantidad completa de producciones total o parcialmente mexicanas, así como datos sobre su producción. 


\section{DISERTACIONES}

Se creó una base de datos con el registro de cada uno de los títulos y el resto de la información solicitada. Esta estrategia permitió encontrar similitudes y diferencias entre las recomendaciones para agruparlas según los datos recabados en la segunda parte. Los datos obtenidos se procesaron con RStudio, entorno integrado para el manejo de comandos de lenguaje de programación R, libre y de código abierto (RStudio Team, 2020).

\section{Resultados}

Los resultados del procesamiento de información se dividen en tres partes. En la primera se trabaja sobre la diversidad de los contenidos ofertados por los resultados de las búsquedas. En la segunda se intentan identificar los factores que orientan distintas recomendaciones para diferentes tipos de audiencias. En la tercera se intenta determinar el nivel de aceptación de las recomendaciones por parte de los usuarios como parte de un proceso de negociación mutua.

\section{Netflix y diversidad audiovisual}

Algunas estimaciones indican que a fines de 2020 México poseía ocho millones de suscriptores y ocupaba el sexto lugar mundial en ganancias dentro de la compañía (Moody, 2020). Esta posición llevó a sus directivos a impulsar las producciones mexicanas dentro de sus títulos originales (Tillman, 2019). A comienzos del 2021, existen 143 producciones total o parcialmente mexicanas en el catálogo de Netflix (Reelgood, 2021a). Una cantidad mucho mayor a los 67 títulos de Brasil (Reelgood, 2021b) y los 63 títulos de Argentina (Reelgood, 2021c). Teniendo en cuenta los 143 títulos identificados, la diversidad de contenidos ofrecida por la plataforma es considerablemente menor. De las tres palabras clave, "México" es la que arroja menos títulos, 31 en total, mientras que "mexicanos" arroja 66 y "mexicanas", 77. Hay que tener en cuenta, sin embargo, que al estudiarse los siete primeros resultados de las listas de 79 consultados, estos 31, 66 y 77 títulos ocuparon 550 posiciones aproximadamente.

En la figura 1 se observan todos los títulos que arrojaron las tres claves de búsqueda. Según la distribución de títulos por posiciones en la lista de recomendación, las primeras tres son ocupadas por pocos títulos recurrentemente recomendados, mientras que las últimas cuatro lo son por muchos títulos poco recomendados, a modo de estructura de cola larga (Anderson, 2009). La personalización, que implica la inclusión de muchos títulos recomendados a pocas personas, se despliega en las últimas posiciones de recomendación antes que en las primeras. En la cabeza, en cambio, los títulos adoptan posiciones fijas, a modo de las grillas televisivas. Este hallazgo coincide con la perspectiva de Jenner (2018), quien afirma que la estructura de Netflix no puede ser vista de manera rígida, pero tampoco enteramente individualizada. Es necesario diferenciar que la grilla televisiva se caracteriza por una clasificación temporal, mientras que las recomendaciones en Netflix siguen un orden en el que los primeros puestos señalan algún tipo de preferencia sobre los siguientes.

Debe agregarse que del mismo modo que las grillas, según confirman varios de los usuarios contactados, las listas de recomendaciones no descuentan los títulos ya vistos. Además, la personalización es menor a la oferta fija de contenidos en todos los casos, especialmente en los resultados de la palabra clave "México". 


\section{DISERTACIONES}

ESTUDIOS

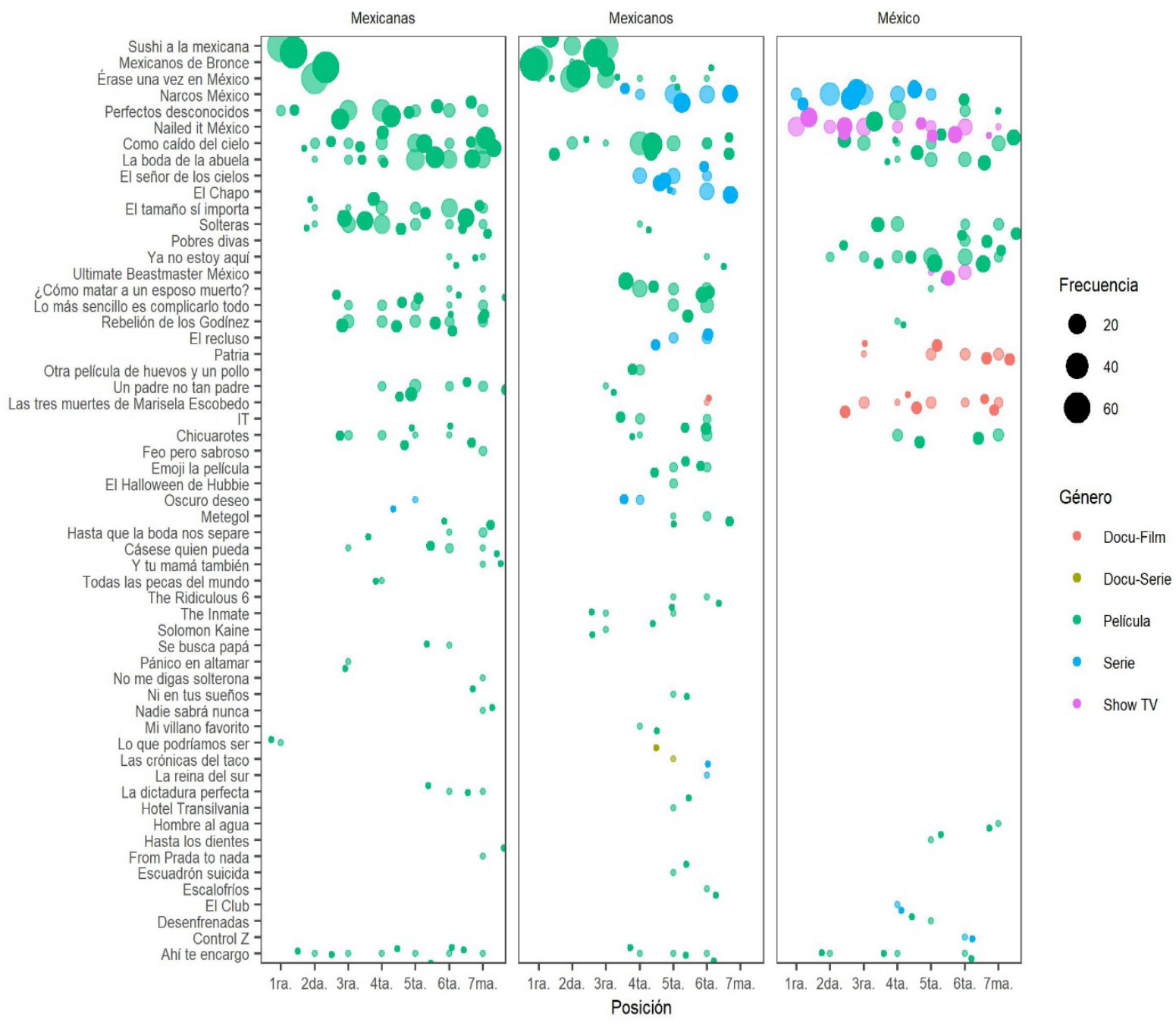

Figura 1. Lista de recomendaciones por palabra clave ordenadas por posición de recomendación

Fuente: elaboración propia, diseñada por: Manuel Cebral.

La figura 1 evidencia también cómo Netflix gestiona la diversidad de formatos. Para la clave de búsqueda "México", más de la mitad de las recomendaciones son películas, $54 \%$, mientras que las series ocupan un $20 \%$, las series y películas documentales otro $20 \%$, y los programas de TV, un $2 \%$. Las series son más recomendadas que las películas en la primera mitad de la lista, mientras que las películas ocupan la segunda mitad. Además, las series 


\section{DISERTACIONES}

ESTUDIOS

Economía de la atención: datos, redes sociales y consumos digitales

ISSN: 1856-9536

Doi: https://doi.org/10.12804/revistas.urosario.edu.co/disertaciones/a.10616

Volumen 15, Número 2 / Julio-diciembre 2022

Versión PDF para imprimir desde

http://revistas.urosario.edu.co/index.php/disertaciones

son bien producciones estadounidenses o coproducciones con México, mientras que entre las películas predominan las producciones locales. Estas ocupan la segunda mitad del gráfico, con una frecuencia de recomendación menor al $30 \%$. La búsqueda con la palabra clave "mexicanas" arroja una sola serie mientras que el resto son películas y con "mexicanos" arroja seis series, una película y una serie documental, mientras que el resto son películas de ficción. Solamente la palabra clave de búsqueda "México" arroja resultados relevantes para documentales en formato película o serie.

Además, la cabeza y la cola indican otra división: los títulos con algún tipo de característica transnacional, sea por su producción, su formato o su narrativa, se encuentran en la cabeza. Por el contrario, la cola se forma en su mayoría con películas de temática y producción local, salvo algunas excepciones. Se infiere que el efecto de cola larga se debe al más arriba mencionado fenómeno de amplificación algorítmica (Sadagopan, 2019), en el que se refuerza la popularidad de los contenidos más populares. En un contexto transnacional, es complejo determinar si estas recomendaciones se ajustan a una diversidad de fuentes, ya que muchos títulos que se identifican con México son realizados por productoras estadounidenses para audiencias globales de habla española. La figura 2 muestra los títulos que aparecen más de diez veces en los resultados de búsqueda de "México" y el origen de su producción. Los títulos más recomendados muestran una presencia protagónica de Estados Unidos, sea como único productor o coproductor con México. A excepción del film Ya no estoy aquí, los contenidos producidos en México no ocupan los primeros lugares en la frecuencia de recomendaciones, aun siendo originales de Netflix.

En esta selección de títulos, la política transnacional de Netflix es visible tanto en el origen de las producciones como en el nivel del contenido. Implica una relación asimétrica con Estados Unidos que se materializa en la coproducción de las series más recomendadas: Narcos México, y en la versión local de programas internacionales como Nailed it México. Además, hay que agregar que Netflix no proporciona dentro de su plataforma información sobre el origen de las producciones, para obtenerla es necesario buscar en sitios externos. Esta omisión contribuye al efecto de catálogo global que la compañía busca producir intencionalmente (Lobato, 2019).

Teniendo en cuenta que en la lista de 31 títulos correspondientes a la palabra clave "México" se incluyen 10 títulos de producción norteamericana, se concluye que el conjunto de recomendaciones de producción mexicana constituye menos del $20 \%$ de las posibilidades de recomendación, las que, como se señaló más arriba, incluyen un total de 143 títulos. Se evidencia entonces que la mayoría del catálogo de producciones mexicanas queda oculto. La información recolectada evidencia que la estrategia de transnacionalización para México depende de relaciones asimétricas con Estados Unidos y que la diversidad de recomendaciones es poca con relación a los contenidos de producción o coproducción mexicana. 


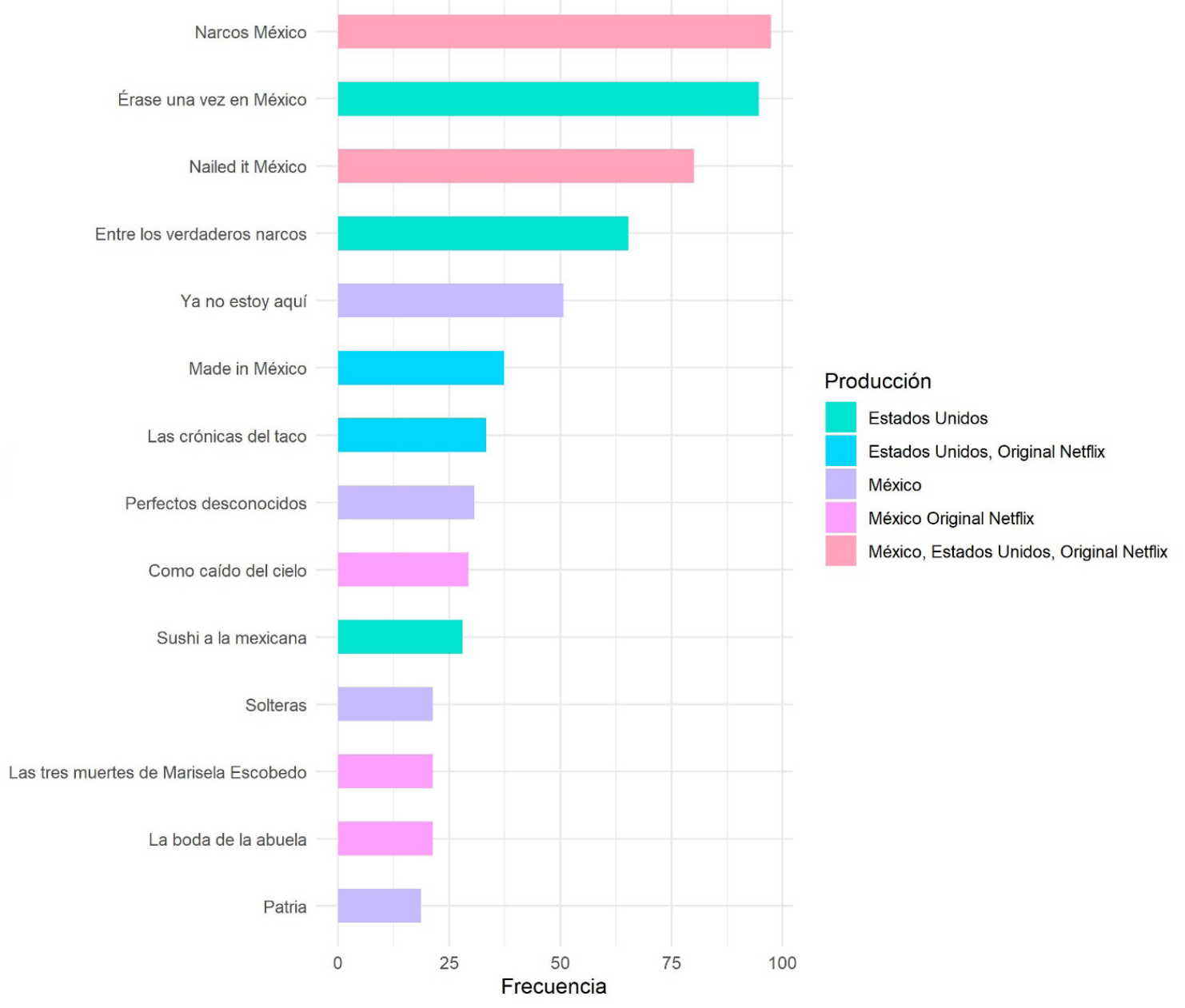

Figura 2. Producciones más frecuentemente recomendadas con la palabra clave de búsqueda "México"(total de recomendaciones de las siete primeras posiciones)

Fuente: elaboración propia con datos extraídos por la plataforma Reelgood.com

Con relación a la diversidad de contenido ofrecida, se observan recurrencias. La temática del narcotráfico se encuentra siempre presente en los primeros puestos de las recomendaciones. Existe, podemos suponer, una asociación relacionada con el éxito internacional de Narcos México y la recurrencia algorítmica de recomendar lo más visto. En el $70 \%$ de los casos, la recomendación que sigue en segundo lugar a Érase una vez en México, es Narcos México. Así, las recomendaciones van formando patrones de reordenamiento de títulos dependiendo de 


\section{DISERTACIONES}

ESTUDIOS

Economía de la atención: datos, redes sociales y consumos digitales

ISSN: 1856-9536

Doi: https://doi.org/10.12804/revistas.urosario.edu.co/disertaciones/a.10616

Volumen 15, Número 2 / Julio-diciembre 2022

Versión PDF para imprimir desde

http://revistas.urosario.edu.co/index.php/disertaciones

la que encabece la recomendación. El binomio que asocia Érase una vez en México con Narcos México, al que en varios casos se agrega la serie documental Entre los verdaderos narcos, se asocia al comportamiento algorítmico identificado por Tufekci (2018), en el que, frecuentemente, seguida a una versión liviana de un contenido se provee una recomendación de esa misma temática más acentuada.

\section{Sistemas de recomendación e información de entrada}

En este apartado se analiza la influencia de los perfiles de usuarios y sus consumos en las recomendaciones automatizadas. Netflix ofrece en su interfaz la creación de varios perfiles dentro de una misma cuenta. Esta distinción interna facilita la elaboración de recomendaciones de acuerdo con los consumos de cada perfil. Sin embargo, dicha personalización no se lleva a la práctica completamente en el uso de la plataforma: muchos usuarios comparten sus perfiles con otros convivientes y personas cercanas, y suelen ver contenidos junto con ellas. Esas personas pueden tener edades, identidades de género y preferencias diferentes entre sí y usar todas el mismo perfil. En el caso de esta muestra, cerca de la mitad de las personas consultadas comparte su perfil con otras de diferente género. Por eso se optó, como se evidencia en la tabla 1, por discriminar entre perfiles femeninos, masculinos y mixtos para considerar los perfiles compartidos con personas del género opuesto.

Tabla 1. Recomendaciones por título y tipo de perfil

\begin{tabular}{|c|c|c|c|}
\hline Título & Perfil masculino & Perfil femenino & Perfil mixto \\
\hline Narcos México & 93 & 100 & 97 \\
\hline Había una vez en México & 100 & 96 & 91 \\
\hline Nailed it México & 36 & 68 & 85 \\
\hline Ya no estoy aquí & 50 & 50 & 45 \\
\hline Dentro de los verdaderos Narcos & 29 & 61 & 52 \\
\hline Made in México & 71 & 36 & 15 \\
\hline Perfectos desconocidos & 21 & 25 & 36 \\
\hline La boda de la abuela & 14 & 21 & 24 \\
\hline Como caído del cielo & 29 & 14 & 15 \\
\hline Las tres muertes de Maricela Escobedo & 21 & 21 & 12 \\
\hline Las crónicas del taco & 35 & 14 & 18 \\
\hline Solteras & 21 & 21 & 3 \\
\hline Sushi a la mexicana & 7 & 18 & 18 \\
\hline Chicuarotes & 21 & 0 & 18 \\
\hline Ahí te encargo & 14 & 11 & 0 \\
\hline El diablo viste a la moda & 14 & 7 & 3 \\
\hline
\end{tabular}




\section{ESTUDIOS}

\section{DISERTACIONES}

Economía de la atención: datos, redes sociales y consumos digitales

ISSN: 1856-9536

Doi: https://doi.org/10.12804/revistas.urosario.edu.co/disertaciones/a.10616

Volumen 15, Número 2 / Julio-diciembre 2022

Versión PoF para imprimir desde

http://revistas.urosario.edu.co/index.php/disertaciones

El Chapo

Patria

\section{7}

7
11

0
6

15

Nota: Los títulos con diferencias significativas de género se marcan en negrita.

Fuente: elaboración propia.

Se vincularon las recomendaciones de títulos y su jerarquización con las variables de género femenino o masculino, edad y lugar de residencia de los usuarios consultados. Algunas recomendaciones son sensibles a la primera variable, mientras que otras se recomiendan equitativamente. En la tabla 1 se resume el análisis de los primeros títulos recomendados en la primera, segunda y tercera posición. El programa Nailed it México, un concurso gastronómico sobre repostería, es más frecuentemente recomendado si la dueña del perfil es mujer, $68 \%$; si lo comparte con varones, sube al $85 \%$. Pero la frecuencia de recomendación cae al $36 \%$ si el dueño del perfil es hombre y no lo comparte con mujeres.

Los títulos que contienen narrativas acerca del narcotráfico son altamente recomendados, sin diferencia de género, solo que en caso de aparecer Nailed it Mexico como primera opción, lo que se da más en el caso de perfiles femeninos, las mencionadas series se desplazan a la segunda y tercera posición de recomendación (Figura 3).
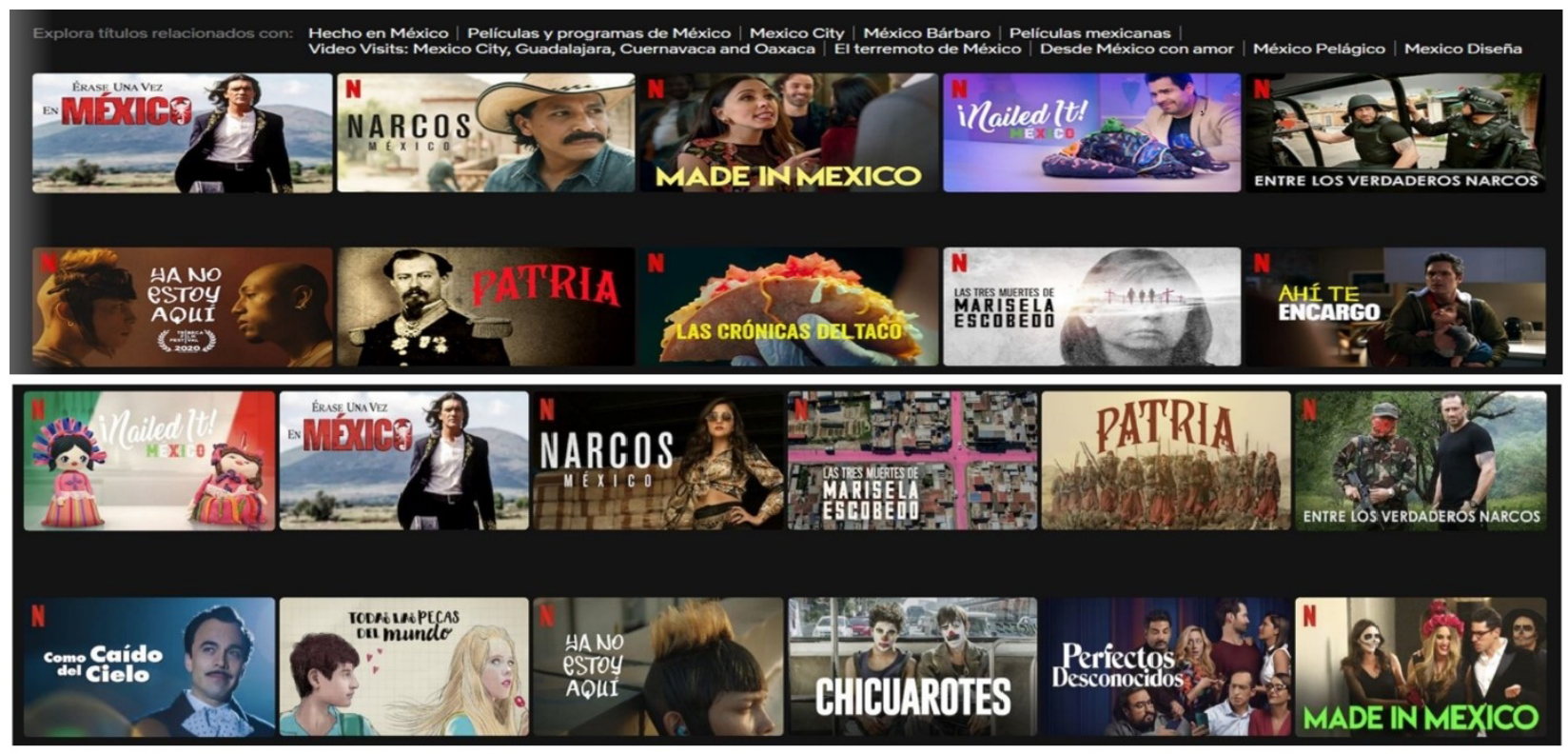

Figura 3. Capturas de pantalla sobre primeros puestos en el orden de recomendaciones con la clave de búsqueda "México"

Nota: Arriba: captura de un consultado. Abajo: captura de una consultada.

Fuente: capturas de pantalla proporcionadas por los consultados. 


\section{DISERTACIONES}

Algunos títulos cuyos protagonistas son varones, como la comedia Como caído del cielo, basada en la figura del actor mexicano Pedro Infante, y Chicuarotes, que narra las desventuras de dos jóvenes varones que llegan a Ciudad de México desde su pueblo natal, son más recomendados a hombres que a mujeres. Sin embargo, el lugar de residencia, dentro o fuera de México, no introduce variaciones en las recomendaciones. En la mayoría de los casos la dupla formada por el film Érase una vez en México y la serie Narcos México, ambas acerca del narcotráfico, ocupan los primeros lugares.

Tampoco se han identificado diferencias sustanciales por edad, a excepción de quienes compartían su perfil con hijas o hijos menores de edad. En esos casos se han identificado títulos infantiles en los resultados de "mexicanas" y “mexicanos", aunque no necesariamente todos los títulos se asocian a México. Este caso es útil para inferir que el sistema de recomendación se alimenta con los consumos de los usuarios, antes que con datos predeterminados de identidad de género o edad.

\section{Usuarios y algoritmos: un proceso de mutua domesticación}

Para determinar la aceptación de los contactos consultados a las recomendaciones de Netflix y cómo esta relación se construye mutuamente, se evaluó la conformidad general de los usuarios con los resultados de las búsquedas y la aceptación de los títulos recomendados más frecuentes. Como contexto, se preguntó a los consultados si habían visto títulos mexicanos en el último año, el $83 \%$ respondió que había visto un poco, el $8,5 \%$ contestó que había visto mucho y el restante $8,5 \%$ contestó que no había visto nada, por lo que puede decirse que la disposición a ver contenidos mexicanos es generalizada.

\section{Tabla 2. Aceptación de los usuarios consultados a los títulos recomendados}

\begin{tabular}{|c|c|c|}
\hline Título & Aceptación & Rechazo \\
\hline Érase una vez en México & 65 & 35 \\
\hline Nailed it México & 62 & 38 \\
\hline Entre los verdaderos narcos & 57 & 43 \\
\hline Mexicanos de bronce & 57 & 43 \\
\hline Perfectos desconocidos & 50 & 50 \\
\hline Como caído del cielo & 51 & 49 \\
\hline Solteras & 41 & 59 \\
\hline Narcos México & 38 & 62 \\
\hline
\end{tabular}

Nota: valores expresados en porcentajes.

Fuente: elaboración propia. 


\section{DISERTACIONES}

Teniendo en cuenta esta amplia disposición, la mitad de los contactados asocia los contenidos ofrecidos por la plataforma con sus consumos, mientras que la otra mitad los asocia muy poco o nada. Quienes vieron mucho contenido mexicano se encuentran en el primer grupo y quienes no vieron nada, en el segundo. El control sobre los principales títulos recomendados arrojó en general una disposición favorable a ver las recomendaciones (tabla 2).

El índice de aceptación, formado por la suma de las recomendaciones vistas y las que podrían verse, se encuentra por encima del $50 \%$ en 6 de los 8 títulos evaluados, mientras que los títulos más recomendados son los más aceptados, con excepción de Solteras y Narcos México.

Siles et al. (2019) evidencian que la relación entre los usuarios y los algoritmos consiste en un proceso de mutua domesticación. Por un lado, los usuarios con sus consumos orientan sus recomendaciones y, por el otro, el algoritmo modela los gustos de los usuarios insistiendo en crear a su usuario ideal. Puede decirse entonces que la plataforma realiza eficientemente la domesticación de los usuarios: es capaz de persuadirlos para que vean sus recomendaciones. Varios de los consultados han señalado que asocian las recomendaciones con otros productos de los mismos géneros narrativos que han visto anteriormente, por lo que este elemento resulta importante en el proceso de domesticación que implica la aceptación de las recomendaciones por parte de los usuarios. El nivel de aceptación concuerda además con la amplificación algorítmica de los contenidos más recomendados señalada más arriba.

Los usuarios resistentes a la domesticación de la plataforma no perciben diversidad en las recomendaciones. Varios de los consultados han expresado su crítica y desinterés hacia las recomendaciones de narco-narrativas, mientras que otros perciben estereotipos en la representación de la identidad mexicana, lo que puede vincularse con el rechazo de la película Solteras. Por último, también identifican que los títulos que más les gustan salen muy abajo en la lista de recomendaciones o que no existen coincidencias entre lo que ven y lo que la plataforma les recomienda.

\section{Discusión}

Este trabajo puso de manifiesto la importancia de los procesos automatizados de recomendación cuando se trata la promoción de la diversidad en las plataformas de video bajo demanda. La comparación de las listas de recomendación con el catálogo de producciones mexicanas identificadas en la base de datos de Reelgood, indica que el total de títulos recomendados es poco diverso en cuanto a contenidos, géneros narrativos y formatos.

La estructura de cola larga (Anderson, 2008) fragmenta las listas de recomendación en dos partes en cuanto a la diversidad de fuentes, contenidos y exposición (García Leiva, 2019). Los primeros puestos de recomendaciones materializan la estrategia transnacional, las producciones estadounidenses y los títulos originales de Netflix, lo que coincide con los hallazgos de Heredia-Ruiz et al. (2021). Dichas producciones priorizan narrativas mexicanas sobre el narcotráfico manufacturadas en Estados Unidos (Orozco Gómez, 2020), y las originales de Netflix, lo que refuerza la histórica relación asimétrica entre México y Estados Unidos en el intercambio de bienes culturales (García Canclini,1996). La base de la clasificación, en cambio, contiene los títulos de producción local, orientados a la personalización y recomendados en menor cantidad. Estos no emergen hasta la quinta posición de recomendaciones. Se necesitan estudios más detallados sobre la composición del catálogo dispo- 


\section{DISERTACIONES}

ESTUDIOS

Economía de la a ter

IISN: 1856-9536

Doi: https://doi.org/10.12804/revistas.urosario.edu.co/disertaciones/a.10616

Volumen 15, Número 2/ Julio-diciembre 2022

Versión pof para imprimir desde

http://revistas.urosario.edu.co/index.php/disertaciones

nible en México para identificar las posibles desventajas comerciales para las productoras locales que proveen de contenidos a la plataforma.

Esta organización sigue la lógica de la amplificación algorítmica, que describe Sadagopan (2019). En los casos de las narrativas sobre el narcotráfico, el orden de las recomendaciones comienza con las más livianas y prosigue con otras más acentuadas con relación al tema tratado, lo que coincide con lo señalado por Tufekci (2018). La lógica de organización de las recomendaciones se encuentra entre la estructura fija de la grilla televisiva y la personalización de contenidos, que confirma lo señalado por Jenner (2018), aunque la distribución de las recomendaciones adopta una forma más estructurada y menos personalizada en el caso de México.

Con relación a los comportamientos algorítmicos (Bandy, 2021), los identificados en este trabajo se sitúan en la categoría de distorsión, ya que en los primeros puestos los resultados son recurrentes, de poca diversidad e invisibilizan gran parte del catálogo mexicano en Netflix.

Las recomendaciones provistas a los consultados emergen de patrones de consumo, no de las variables demográficas como dato de entrada del algoritmo. Si existen diferencias de género se deben a consumos previos diferenciados. Respecto a la aceptación de dichas recomendaciones, la priorización de narrativas relacionadas con el narcotráfico representa el punto de mayor resistencia de la mitad de la audiencia consultada acerca de la aceptación de los contenidos recomendados, aunque la otra mitad consume y disfruta este tipo de contenidos. La división entre quienes consumen y critican las narrativas del narcotráfico, y la insistencia en las recomendaciones evidencia los procesos de domesticación por parte de la plataforma: se trata de modelar los consumos de la audiencia persuadiéndola de visionar sus recomendaciones, según señalan Siles et al. (2019). A la vez, y siguiendo la línea de los autores, el uso colectivo de perfiles individuales puede significarse como una resistencia de los usuarios a subordinarse a las prácticas individualizantes de la compañía. El visionado colectivo vuelve menos preciso el algoritmo y se rebela contra el diseño de la interfaz, dejando lugar a prácticas propias que no dependen de las estrategias de mercadotecnia de la plataforma ni de sus disposiciones técnicas.

\section{Conclusiones}

A través de las recomendaciones automáticas emerge una geografía mediática mexicana fragmentada en dos espacios. El primero es un espacio transnacional tanto en el nivel de la producción como en el de los contenidos, que ocupa las primeras posiciones de las recomendaciones y sus títulos son los más recurrentes. La temática del narcotráfico se prioriza en ese espacio. Esta consolidación de las narco-narrativas consolida una visión que refuerza la identificación de México con el narcotráfico, que debe ser estudiada con mayor profundidad. El segundo es un espacio local, construido principalmente a través de películas. Las más recomendadas para consumo masivo suelen ser las comedias.

Los funcionamientos algorítmicos descritos en este trabajo ponen de manifiesto las históricas relaciones desiguales entre México y Estados Unidos y la construcción de una geografía mediática basada en estereotipos. Así se confirma la hipótesis de Hallinan y Striphas (2016) acerca de la activa intervención de los algoritmos en la producción de la cultura y del sentido, y añade aspectos culturales y políticos a la visión sociotécnica de los algoritmos descrita por Rieder (2020). También, agrega que una regulación integral debería contemplar, además de las cuotas de contenido ya señaladas por lordache (2021), auditorías sobre los procesos automati- 


\section{DISERTACIONES}

ESTUDIOS

zados para que estos garanticen la diversidad de contenidos ofertada (Ranavoison, 2019) y la visibilidad a las producciones locales.

La comprensión de las lógicas automatizadas que guían a los usuarios a tomar sus decisiones es relevante para el estudio de la diversidad audiovisual de las plataformas. Sin embargo, su caracterización presenta un gran desafío para los estudios de medios, debido a la complejidad de las variables y el secreto de sus procesos; los métodos de auditoría algorítmica se presentan como una herramienta eficaz para desentrañar parcialmente los funcionamientos algorítmicos. Dado que las plataformas no aportan información sobre los procesos, resulta fundamental el contacto que los investigadores puedan establecer con clientes de las plataformas para desentrañar los procesos algorítmicos. Sin embargo, las auditorías algorítmicas tienen sus limitaciones, dado que si bien expresan la vinculación de los algoritmos y sus usuarios, el método es inferencial y válido solo para el conjunto de datos analizados. Posteriores estudios, por ejemplo con relación a geografías mediáticas producidas en otras localizaciones, podrían contribuir a verificar su eficiencia.

México ocupa un lugar interesante en la estrategia transnacional de Netflix para la producción y difusión de contenidos, pero si las producciones mexicanas quedan invisibilizadas en los primeros puestos de los sistemas de recomendaciones, esa posición queda en desventaja. Por otro lado, es sabido que en ese catálogo se encuentran películas y series de alta calidad que no se verifican en las recomendaciones estudiadas en esta muestra. La composición del catálogo mexicano de Netflix debe ser estudiada en profundidad a futuro, así como la exposición a patrones individuales de consumo de contenidos relativos a México. Asimismo, las lógicas de resistencia a las recomendaciones algorítmicas evidenciadas en esta y precedentes investigaciones puede dar lugar al estudio de las geografías mexicanas en Netflix no ya construida por los sistemas automatizados, sino por las prácticas mediáticas de los usuarios.

\section{Referencias}

1. Albornoz, L. A., \& García Leiva, M. T. (2017). Diversidad e industrias audiovisuales: El desafío cultural del siglo xxı. Fondo de Cultura Económica.

2. Anderson, C. (2009). The long tail: Why the future of business is selling less of more. Random House Business.

3. Bandy, J. (2021). Problematic machine behavior: A systematic literature review of algorithm audits. Proceedings of the ACM on human-computer interaction, 5(cscw1), 74:1-34. https://doi.org/10.1145/3449148

4. Bucher, T. (2018). If ... then: Algorithmic power and politics. Oxford University Press.

5. Cybenko, G., \& Brewington, B. (1999). The foundations of information push and pull. En G. Cybenko, D. P. O'Leary, \& J. Rissanen (Eds.), The mathematics of information coding, extraction and distribution (pp. 9-30). Springer. https://doi.org/10.1007/978-1-4612-1524-0_2

6. Hallinan, B., \& Striphas, T. (2016). Recommended for you: The Netflix Prize and the production of algorithmic culture. New Media \& Society, 18(1), 117-137. https://doi.org/10.1177/1461444814538646

7. Heredia-Ruiz, V., Quirós-Ramírez, A. C., \& Quiceno-Castañeda, B. E. (2021). Netflix: Catálogo de contenido y flujo televisivo en tiempos de big data. Revista de Comunicación, 20(1), 117-136. https://doi.org/10.26441/ RC20.1-2021-A7 


\section{DISERTACIONES}

ESTUDIOS

Economía de la atención: datos, redes sociales y consumos digitales

ISSN: 1856-9536

Doi: https://doi.org/10.12804/revistas.urosario.edu.co/disertaciones/a.10616

Volumen 15, Número 2 / Julio-diciembre 2022

Versión PDF para imprimir desde

http://revistas.urosario.edu.co/index.php/disertaciones

8. García Canclini, N. (1996). Políticas culturales e integración norteamericana: Una perspectiva desde México. En Autor (Ed.), Culturas en globalización América Latina, Europa, Estados Unidos: Libre comercio e integración (pp. 13-41). cncA, Clacso, Nueva Sociedad.

9. García Leiva, M. T. (2019). Plataformas en línea y diversidad audiovisual: Desafíos para el mercado español. cIc: Cuadernos de Información y Comunicación, 24, 73-93. https://doi.org/10.5209/ciyc.64639

10. Gomez-Uribe, C. A., \& Hunt, N. (2016). The Netflix recommender system: Algorithms, business value, and innovation. ACM Transactions on Management Information Systems, 6(4), 13:1-19. https://doi. org $/ 10.1145 / 2843948$

11. Iordache, C. (2021). Netflix in Europe: Four markets, four platforms? A comparative analysis of audio-visual offerings and investment strategies in four Eu states. Television \& New Media. https://doi. org/10.1177/15274764211014580

12. Jenner, M. (2018). Netflix and the re-invention of television. Springer International Publishing AG.

13. Lobato, R. (2017). Rethinking international tv flows research in the age of Netflix. Television \& New Media, 19(3), 241-256. https://doi.org/10.1177/1527476417708245

14. Lobato, R. (2019). Netflix nations: The geography of digital distribution. nYu Press.

15. Lozano, J. C. (2006). Diversidad cultural y televisión en México. Comunicación y Sociedad, 5, 137-156. https://doi.org/10.32870/cys.v0i5.4016

16. Moody, R. (2020). Netflix subscribers and revenue by country [2020 version]. https://www.comparitech. $\mathrm{com} /$ tv-streaming/netflix-subscribers/

17. Netflix Research. (s. f.). Personalization and search. Helping members discover content they'll love. https:// research.netflix.com/business-area/personalization-and-search

18. Plummer, L. (2017). This is how Netflix's top-secret recommendation system works. Wired uk. https:// www.wired.co.uk/article/how-do-netflixs-algorithms-work-machine-learning-helps-to-predict-whatviewers-will-like

19. Orozco Gómez, G. (2020). La ficción es otra forma de contar la realidad. En Autor, Televisión en tiempos de Netflix. Una nueva oferta mediática (pp. 11-17). Universidad de Guadalajara.

20. Padilla de la Torre, R. (2012) Geografías ciudadanas ymediáticas. Universidad Autónoma de Aguascalientes.

21. Piñón, F. (2020). Un reconocimiento de la infraestructura de la red de internet para servicios de VoD en Latinoamérica. En G. Orozco Gómez (Ed.), Televisión en tiempos de Netflix. Una nueva oferta mediática (pp. 17-46). Universidad de Guadalajara.

22. Ranavoison, H. (2019). Online platforms and cultural diversity in the audiovisual sectors: A combined look at concentration and algorithms. En L. Albornoz \& M. T. García Leiva (Eds.), Audiovisual industries and diversity. Economics and policies in the digital era (pp. 100-118). Routledge. https://doi. org/10.4324/9780429427534-6

23. Reelgood (2021a) Full list of Mexican movies and tv shows on Netflix. https://reelgood.com/origin/mexico/ on-netflix

24. Reelgood (2021b) Full list of Brazilian movies and tv shows on Netflix. https://reelgood.com/origin/brazil/ on-netflix 


\section{DISERTACIONES}

25. Reelgood (2021c) Full list of Argentinian movies and TV shows on Netflix. https://reelgood.com/origin/ argentina/on-netflix

26. Rieder, B. (2020). Engines of order a mechanology of algorithmic techniques. Amsterdam University Press. https://doi.org/10.2307/j.ctv12sdvf1

27. Rieder, B., Matamoros-Fernández, A., \& Coromina, O. (2018). From ranking algorithms to 'ranking cultures': Investigating the modulation of visibility in YouTube search results. Convergence, 24(1), 50-68. https://doi. org/10.1177/1354856517736982

28. RStudio Team. (2020). RStudio: Integrated Development for R. RStudio. http://www.rstudio.com/

29. Sadagopan, S. M. (2019). Feedback loops and echo chambers: How algorithms amplify viewpoints. The Conversation. http://theconversation.com/feedback-loops-and-echo-chambers-how-algorithms-amplify-viewpoints-107935

30. Sandvig, C., Hamilton, K., Karahalios, K., \& Langbort, C. (2014). Auditing algorithms: Research methods for detecting discrimination on internet platforms. "Data and discrimination: Converting critical concerns into productive inquiry." Ponencia presentada en la sexagésima cuarta Annual Meeting of the International Communication Association, Seattle, WA.

31. Siles, I., Espinoza-Rojas, J., Naranjo, A., \& Tristán, M. F. (2019). The mutual domestication of users and algorithmic recommendations on Netflix. Communication, Culture and Critique, 12(4), 499-518. https:// doi.org/10.1093/ccc/tcz025

32. Tillman, L. (2019). Netflix, drawn to talent, boosts production in Mexico with 50 films and series. Los Angeles Times. https://www.latimes.com/business/hollywood/la-fi-ct-netflix-mexico-20190319-story.html

33. Tufekci, Z. (2018). YouTube, the Great Radicalizer. The New York Times. https://www.nytimes. com/2018/03/10/opinion/sunday/youtube-politics-radical.html 